Maria Inês Couto de Oliveira'

Kátia Silveira da Silva"

Saint Clair Gomes Junior"

Vânia Matos Fonseca',!I

\section{Resultado do teste rápido anti- HIV após o parto: uma ameaça à amamentação ao nascimento}

\author{
Delivering rapid HIV tests \\ results after delivery: a threat to \\ breastfeeding at birth
}

Departamento de Epidemiologia e Bioestatística. Instituto de Saúde da Comunidade. Universidade Federal Fluminense. Niterói, RJ, Brasil

Unidade de Pesquisa Clínica. Instituto Fernandes Figueira. Fundação Oswaldo Cruz. Rio de Janeiro, RJ, Brasil

Correspondência | Correspondence: Maria Inês Couto de Oliveira

R. Marquês do Paraná, 303/3º andar - Centro

24033-900 Niterói, RJ, Brasil

E-mail: marinesco@superig.com.br

\section{RESUMO}

OBJETIVO: Analisar fatores associados à não-amamentação na primeira hora de vida, sobretudo a influência do momento do resultado do teste rápido anti-HIV.

MÉTODOS: Estudo de coorte, sendo o ponto inicial a submissão ao teste rápido e o final a primeira mamada do bebê. A população estudada incluiu 944 parturientes submetidas ao teste rápido anti-HIV, com resultado negativo, em 2006, nos cinco hospitais amigos da criança do Sistema de Gestação de Alto Risco no município do Rio de Janeiro, RJ. Entrevistadoras treinadas obtiveram dados do laboratório e do prontuário e no pós-parto aplicaram questionário para entrevista às mães. O modelo multinível foi adotado para analisar a influência de características sociodemográficas, de assistência pré-natal e ao parto sobre a não-amamentação na primeira hora de vida.

RESULTADOS: Dentre as participantes, apenas 15,6\% receberam seu resultado antes do parto, $30,8 \%$ depois do parto e $53,6 \%$ ainda desconheciam o resultado ao ser entrevistada. A prevalência de não-amamentação na primeira hora de vida foi de 52,5\% (IC 95\%: 49,3;55,8). Após ajuste, o recebimento do resultado do teste rápido após o parto dobrou o risco da não-amamentação na primeira hora de vida ( $R R=2,06$; IC 95\%: 1,55;2,75). Outros fatores de risco foram: cor não branca, renda materna de um salário mínimo ou menos, parto cesáreo, mãe não querer amamentar o bebê ao nascimento e mãe referir que a equipe hospitalar não a escutava. $\mathrm{O}$ desconhecimento da realização do teste rápido anti-HIV pela mãe se mostrou como fator de proteção.

CONCLUSÕES: O principal fator de risco para a não-amamentação na primeira hora de vida foi o recebimento do resultado do teste rápido após o parto. O teste anti-HIV deve ser amplamente disponibilizado no pré-natal e o teste rápido deve ser realizado sob indicação, na admissão, com busca ativa e pronta comunicação do resultado à mulher.

DESCRITORES: Aleitamento Materno. Mulher. Sorodiagnóstico da AIDS. Transmissão Vertical de Doença Infecciosa, prevenção \& controle. Estudos de Coortes. 


\begin{abstract}
OBJECTIVE: To analyze factors associated with failure to breastfeed during the first hour of life, especially the influence of time of delivery of rapid HIV test results.

METHODS: Cohort study, beginning with the administration of the rapid test and ending the first time the baby is breastfed. The study population included 944 delivering mothers that received rapid HIV testing with a negative result in five Baby-Friendly hospitals of the High-Risk Pregnancy System in the city of Rio de Janeiro, Southeastern Brazil, in 2006. Trained interviewers obtained data from laboratory and patient charts and interviewed mothers shortly after delivery. The influence of sociodemographic variables and antenatal and delivery care characteristics on failure to breastfeed during the first hour of life was determined through a multilevel model.
\end{abstract}

RESULTS: Among participants, $15.6 \%$ received the result of rapid HIV testing before delivery, $30.8 \%$ after delivery, and $53.6 \%$ had not yet been informed of their results at the time of the interview. Prevalence of failure to breastfeed in the first hour of life was 52.5\% (95\% CI: 49.3;55.8). After adjustment, having received the result of rapid testing only after delivery doubled the risk of failing to breastfeed in the first hour ( $\mathrm{RR}=2.06 ; 95 \% \mathrm{CI}: 1.55 ; 2.75)$. Other risk factors included nonwhite skin color, maternal income of up to one minimum wage, delivery by C-section, mother's lack of desire to breastfeed at birth, and mother's report that the hospital staff did not listen to her. Lack of knowledge of HIV testing from the mother's part was found to be a protective factor.

CONCLUSIONS: The major risk factor for not breastfeeding in the first hour of life was failure to receive the results of rapid HIV testing prior to delivery. HIV testing should be made widely available during antenatal care; rapid testing should be performed upon admission, only when indicated, and with active search and prompt delivery of results to expecting mothers.

DESCRIPTORS: Breast Feeding. Women. AIDS Serodiagnosis. HIV Infections, diagnosis. Infectious Disease Transmission, Vertical, prevention \& control. Cohort Studies.

\section{INTRODUÇÃO}

A amamentação contribui para a saúde da $\operatorname{mulher}^{10} \mathrm{e}$ da criança. ${ }^{21}$ Fatores como falta de apoio à amamentação na atenção primária à saúde, ${ }^{4}$ separação mãe-filho no pós-parto imediato, início tardio da primeira sucção e uso inadequado de suplementos têm contribuído para o desmame precoce. ${ }^{13}$

A Iniciativa Hospital Amigo da Criança (Organização Mundial da Saúde/Fundo das Nações Unidas para a Infância) propõe a mobilização dos serviços obstétricos para a adoção de "Dez Passos para o Sucesso do Aleitamento Materno". ${ }^{17}$ O Passo 4 recomenda: "ajudar as mães a iniciar a amamentação na primeira meia hora após o parto". ${ }^{22}$ Esta ajuda deve ser proporcionada pela equipe de saúde na sala de parto, enquanto mãe e recém- nascido estão alertas e interagindo. $\mathrm{O}$ contato pele a pele propicia a primeira sucção de forma espontânea na primeira hora de vida, ${ }^{16} \mathrm{o}$ que contribui para o aumento da duração do aleitamento materno ${ }^{15}$ e para a redução da mortalidade neonatal. ${ }^{8}$

Apesar das vantagens do aleitamento materno, a sua prática por mães soropositivas é contra-indicada no Brasil, ${ }^{\text {a }}$ pois representa risco adicional de transmissão vertical de 14\% (IC 95\%: 7;22). ${ }^{7}$ A taxa de transmissão vertical do HIV sem qualquer intervenção situa-se em torno de $20 \%$ e, com o uso combinado do Protocolo 076 do Aids Clinical Trial Group (PACTG 076), há

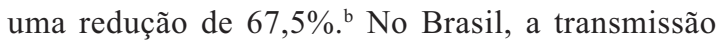
vertical diminuiu de $16,0 \%{ }^{19}$ para $3,7 \%{ }^{5}$ entre 1997 e

a Ministério da Saúde. Secretaria de Assistência à Saúde - PN DST/AIDS. Aleitamento x mulheres infectadas pelo HIV- recomendações. Brasília; 1995.

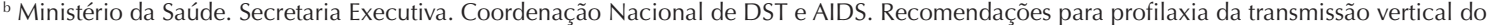
HIV e terapia antiretroviral em gestantes. Brasília; 2003. (Manuais, 46). 
2002, embora a implementação deste protocolo ainda não esteja plenamente concluída.

A ausência de detecção da infecção pelo HIV durante o pré-natal representa uma oportunidade perdida de prevenção da transmissão vertical, e o uso do teste rápido para a detecção de anticorpos anti-HIV no momento da internação para o parto deveria ser uma exceção. Estudo realizado no Rio de Janeiro encontrou uma proporção de $74,7 \%$ de gestantes testadas para o HIV. ${ }^{20}$ A prevalência de mulheres com resultado disponível de exame anti-HIV antes da internação para o parto foi estimada em $62,5 \%$ para o Brasil, e em 78,3\% para a Região Sudeste em 2004. ${ }^{18}$

O Projeto Nascer-Maternidades, ${ }^{a}$ instituído em 2002, visou reduzir a transmissão vertical do HIV por meio de medidas como o estabelecimento do status sorológico de $100 \%$ das parturientes que não tivessem sido testadas durante o pré-natal, mediante a testagem para o HIV no período do pré-parto imediato. ${ }^{3}$ Este teste é voluntário e sigiloso e deve ser realizado mediante aconselhamento e consentimento verbal da mulher. ${ }^{\mathrm{c}}$

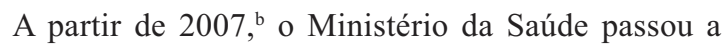
recomendar a oferta de um novo teste para o HIV no terceiro trimestre de gestação para as gestantes testadas no primeiro trimestre. Manteve a recomendação que o profissional de saúde não deva proibir o aleitamento materno no caso de a mulher não ter sido testada ou do resultado do teste não estar disponível, pois a contraindicação deve ser baseada em diagnóstico de HIV reagente e, não havendo este diagnóstico, o aleitamento materno deve ser assegurado a todas as crianças. ${ }^{c}$

A prevalência de soropositividade para o HIV encontrada em 2007 em gestantes no Estado do Rio de Janeiro foi de $0,15 \%$, a menor da Região Sudeste e inferior à prevalência nacional $(0,21 \%) .{ }^{14}$ Mesmo na clientela de gestantes nunca testadas na gravidez e submetidas ao teste rápido, não ultrapassou 3,2\% em estudo realizado em Ribeirão Preto, SP. ${ }^{6}$ Seria esperado, portanto, que a realização do teste rápido não gerasse um impacto importante na amamentação ao nascimento.

O presente estudo teve por objetivo analisar fatores associados à não-amamentação na primeira hora de vida, sobretudo a influência do momento do resultado do teste rápido anti-HIV.

\section{MÉTODOS}

Estudo de coorte realizado no município do Rio de Janeiro, RJ. A população foi composta por todas as mulheres que realizaram teste rápido anti-HIV por ocasião do parto nos cinco Hospitais Amigos da Criança, pertencentes ao Sistema de Gestação de Alto Risco do Sistema Único de Saúde, entre 11 de setembro e 11 de dezembro de 2006. O acompanhamento dessas mulheres iniciou-se na submissão ao teste rápido anti-HIV, recuperado do livro de registros do laboratório, passando pelo momento do parto até o momento da primeira mamada.

O estudo piloto realizado para cálculo amostral indicou uma prevalência de não-amamentação ao nascimento de $50 \%$ entre as mulheres soronegativas submetidas ao teste rápido anti-HIV. A partir deste parâmetro, estimou-se que seria necessária uma amostra de 700 mães para um poder estatístico de $99 \%$ e um nível de confiança de $95 \%$.

Foram incluídas no estudo as mulheres submetidas ao teste rápido anti-HIV, com filhos nascidos vivos, em regime de alojamento conjunto. Foram excluídas aquelas cujos filhos nasceram com Apgar menor do que 7 no quinto minuto e/ou que ficaram em unidade neonatal por qualquer período de tempo, pois estas situações poderiam contra-indicar a amamentação ao nascimento. As parturientes soropositivas também foram excluídas da análise do desfecho não-amamentação na primeira hora de vida.

Para identificação das parturientes submetidas ao teste rápido anti-HIV, foi realizado um levantamento diário no laboratório de cada hospital, sendo o nome destas mulheres transcrito para o formulário de coleta de dados do laboratório. No posto de enfermagem do alojamento conjunto, o formulário era confrontado diariamente com a relação de pacientes internadas. O prontuário destas mães era consultado, os dados relativos ao parto eram transcritos para um mapa diário e as mães elegíveis eram então entrevistadas no alojamento conjunto. Caso no momento da entrevista o recém-nascido ainda não tivesse sido amamentado, a entrevistadora retornava àquela mãe ao final do dia para a coleta do dado referente ao tempo decorrido entre o nascimento e a primeira mamada. Os horários das rotinas do hospital eram utilizados para auxiliar as mães neste recordatório.

Os instrumentos de coleta de dados, previamente testados, foram: formulário para coleta de dados do laboratório do hospital, mapa diário para verificação dos critérios de elegibilidade e transcrição de dados do prontuário materno e questionário para entrevista.

a Ministério da Saúde. Portaria GM n 2104, de 19 de novembro de 2002. Institui no âmbito do Sistema Único de Saúde - SUS o Projeto Nascer-Maternidades. Diario Oficial Uniao. 2002 set 21;Seção1:48.

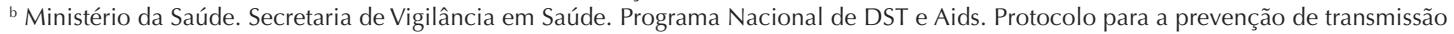
vertical de HIV e sífilis: manual de bolso. Brasília; 2007. (B. Texto Básicos de Saúde -Manuais, 80).

c Ministério da Saúde. Secretaria de Vigilância em Saúde. Secretaria de Atenção à Saúde. Manual normativo para profissionais de saúde de maternidades da iniciativa Hospital Amigo da Criança. Referência para mulheres HIV positivas e outras que não podem amamentar. Brasília; 2004 
As mulheres foram entrevistadas por seis enfermeiras ou acadêmicas de enfermagem treinadas. Cada entrevistadora foi alocada em um hospital e era supervisionada semanalmente por um pesquisador específico, e quinzenalmente pela coordenadora da pesquisa. Uma das entrevistadoras realizava substituições eventuais.

Para comparação do perfil do grupo de mães elegíveis entrevistadas e não entrevistadas, foram colhidos dados das declarações de nascidos vivos dos recémnascidos.

No período do estudo ocorreram 4.895 partos, tendo $1.396(28,5 \%)$ parturientes sido submetidas ao teste rápido anti-HIV. Desse universo, 322 mulheres (23,1\%) foram excluídas, restando 1.074 mães elegíveis. Das elegíveis, 42 mulheres $(3,9 \%)$ se recusaram a participar da pesquisa e houve perda de 77 mulheres $(7,2 \%)$ por alta hospitalar precoce ou outro motivo, sendo entrevistadas 955 parturientes. As características sociodemográficas, de assistência pré-natal e ao parto das mulheres entrevistadas se mostraram semelhantes às das perdas/recusas. A única variável que diferiu $(\mathrm{p}=0,01)$ entre os grupos foi o status prévio de HIV segundo o prontuário.

Para análise dos dados foram utilizados os programas Epi Info e SPSS.

A associação entre a variável de exposição principal "momento do resultado do teste rápido anti-HIV em relação ao parto" e o desfecho "não-amamentação na primeira hora de vida" foi analisada a partir do risco relativo, considerando características: 1- socioeconômicas: idade, escolaridade, raça, trabalho, renda e presença de companheiro; 2- reprodutivas: paridade, número de relacionamentos na vida e no último ano; 3de assistência pré-natal: número de consultas, período de realização de teste anti-HIV na gravidez e seu resultado; 4- de assistência ao parto: mãe ter conhecimento da realização do teste rápido anti-HIV, resultado do teste rápido anti-HIV, tipo de parto, mãe querer colocar o bebê ao peito ao nascimento, mãe considerar que a equipe hospitalar escutava o que ela tinha a dizer sobre si e o bebê, e hospital de ocorrência do parto.

O tempo até a primeira mamada foi categorizado em amamentação na primeira hora de vida ou não-amamentação na primeira hora de vida. O risco relativo, com seu respectivo intervalo com $95 \%$ de confiança, foi estimado a partir de um modelo multinível, com função de ligação complementar $\log \log .{ }^{9}$ Esta função de ligação é preferida para eventos binários com probabilidade de ocorrência muito freqüente, como foi observado. ${ }^{12} \mathrm{~A}$ abordagem multinível foi adotada para captar a influência da correlação intra-hospitalar sobre a não-amamentação na primeira hora de vida. ${ }^{9}$ O modelo tem como variáveis do nível mais agregado as características da unidade e como nível individual as características maternas. As variáveis preditoras do modelo foram definidas após análise bivariada, devendo atingir um nível de significância de 20\% para serem consideradas no processo de modelagem. No modelo final, foram consideradas variáveis com nível de significância menor que 5\%.

O estudo foi aprovado pelos Comitês de Ética do Instituto Fernandes Figueira/Fundação Oswaldo Cruz e da Secretaria Municipal de Saúde do Rio de Janeiro. Os dados foram colhidos mediante assinatura de termo de consentimento livre e esclarecido.

\section{RESULTADOS}

Foi encontrada uma prevalência de soropositividade ao teste rápido anti-HIV de $0,8 \%$, tanto entre o total de parturientes elegíveis e não elegíveis submetidas ao teste (11/1396), quanto entre as mães entrevistadas (8/955). Nenhuma das oito mães soropositivas entrevistadas amamentou seu bebê durante a internação hospitalar, e houve três casos de mães soronegativas em que não foi possível estabelecer o momento da primeira mamada, tendo a análise do desfecho sido realizada em 944 mães. A prevalência de não-amamentação na primeira hora de vida foi de 52,5\% (IC 95\%: 49,3;55,8).

A Tabela 1 mostra que essas mulheres submetidas ao teste rápido soronegativas para o HIV eram jovens, a maioria de cor não-branca e com ensino fundamental completo. Quase metade (46,9\%) das mães trabalhavam fora de casa na época da entrevista ou quando ficaram grávidas. Apenas 27,6\% das mães recebiam mais de um salário mínimo por mês. A renda familiar per capita, em $84,4 \%$ dos domicílios, foi de um salário mínimo ou menos. A grande maioria das mães tinha companheiro, das quais $66,3 \%$ tiveram outros relacionamentos na vida, e entre estas, $13,4 \%$ o tiveram no último ano (dados não mostrados na tabela). Na análise bivariada, a mãe receber um salário mínimo ou menos e ser de cor-não branca se mostraram fatores de risco para a não-amamentação na primeira hora.

A gravidez atual foi planejada em 31,6\% dos casos e $8,8 \%$ das mães não fizeram pré-natal. A maioria $(78,7 \%)$ das mães informou haver realizado exame de rastreamento do HIV no pré-natal, das quais $79,8 \%$ receberam resultado negativo e $20,2 \%$ desconheciam o resultado. Nenhuma destas características esteve significativamente associada ao desfecho (Tabela 2).

Em relação à assistência hospitalar, observou-se que $75,0 \%$ das mães tinham conhecimento da realização do teste rápido anti-HIV pelo hospital. Ao serem perguntadas quando souberam o resultado do teste rápido, apenas $15,6 \%$ havia recebido o resultado antes do parto, $30,8 \%$ recebera depois do parto e mais da metade $(53,6 \%)$ ainda desconhecia o resultado no momento da entrevista (Tabela 3). A proporção de indeterminação do momento do resultado segundo o prontuário materno foi semelhante, de $51,5 \%$. 
Tabela 1. Características socioeconômicas e reprodutivas de mães submetidas ao teste rápido anti-HIV em Hospitais Amigos da Criança segundo análise bivariada em relação à não amamentação do recém-nascido na primeira hora de vida. Rio de Janeiro, RJ, 2006.

\begin{tabular}{|c|c|c|c|c|c|}
\hline \multirow{2}{*}{ Variável } & \multicolumn{2}{|c|}{ Freqüência } & \multicolumn{3}{|c|}{ Não amamentação na primeira hora } \\
\hline & $\mathrm{n}$ & $\%$ & $\%$ & $\mathrm{RR}$ & IC 95\% \\
\hline \multicolumn{6}{|l|}{ Faixa etária (anos) $(\mathrm{n}=944)$} \\
\hline 13 a 19 & 253 & 26,8 & 49,4 & 0,92 & 0,$80 ; 1,06$ \\
\hline 20 a 34 & 607 & 64,3 & 53,7 & 1 & \\
\hline 35 a 45 & 84 & 8,9 & 53,6 & 1,00 & 0,$81 ; 1,23$ \\
\hline \multicolumn{6}{|l|}{ Escolaridade $(n=944)$} \\
\hline Ensino fundamental incompleto & 406 & 43,0 & 51,5 & 1 & \\
\hline Ensino fundamental completo ou mais & 538 & 57,0 & 53,3 & 1,04 & 0,$92 ; 1,18$ \\
\hline \multicolumn{6}{|l|}{ Cor da pele $(n=944)$} \\
\hline Branca & 261 & 27,6 & 43,7 & 1 & \\
\hline Não branca & 683 & 72,4 & 55,9 & 1,28 & 1,$10 ; 1,49$ \\
\hline \multicolumn{6}{|l|}{ Trabalho atual ou quando engravidou $(n=944)$} \\
\hline Sim & 443 & 46,9 & 52,6 & 1 & \\
\hline Não & 501 & 53,1 & 52,5 & 0,998 & 0,$88 ; 1,13$ \\
\hline \multicolumn{6}{|l|}{ Renda materna $(\mathrm{n}=944)$} \\
\hline Mãe não tem renda & 423 & 44,8 & 53,0 & 1,13 & 0,$97 ; 1,31$ \\
\hline Um salário mínimo ou menos & 260 & 27,5 & 58,1 & 1,25 & 1,$06 ; 1,48$ \\
\hline Mais que um salário mínimo & 261 & 27,6 & 46,4 & 1 & \\
\hline \multicolumn{6}{|l|}{ Renda familiar per capita $(\mathrm{n}=877)$} \\
\hline Um salário mínimo ou menos & 739 & 84,4 & 54,0 & 1 & \\
\hline Mais que um salário mínimo & 138 & 15,6 & 48,6 & 0,90 & 0,$75 ; 1,08$ \\
\hline \multicolumn{6}{|l|}{ Tem companheiro $(n=944)$} \\
\hline Sim & 826 & 87,5 & 52,3 & 1 & \\
\hline Não & 118 & 12,5 & 54,2 & 1,04 & 0,$87 ; 1,24$ \\
\hline \multicolumn{6}{|l|}{ Paridade $(\mathrm{n}=939)$} \\
\hline Primípara & 372 & 39,6 & 51,3 & 0,92 & 0,$69 ; 1,23$ \\
\hline 2 a 3 filhos & 444 & 47,3 & 53,4 & 0,99 & 0,$83 ; 1,20$ \\
\hline 4 filhos ou mais & 123 & 13,1 & 53,7 & 1 & \\
\hline
\end{tabular}

Tabela 2. Características de assistência pré-natal de mães submetidas ao teste rápido anti-HIV em Hospitais Amigos da Criança segundo análise bivariada em relação à não amamentação do recém-nascido na primeira hora de vida, Rio de Janeiro, RJ, 2006.

\begin{tabular}{|c|c|c|c|c|c|}
\hline \multirow{2}{*}{ Variável } & \multicolumn{2}{|c|}{ Freqüência } & \multicolumn{3}{|c|}{ Não amamentação na primeira hora } \\
\hline & $\mathrm{n}$ & $\%$ & $\%$ & RR & IC 95\% \\
\hline \multicolumn{6}{|c|}{ Número de consultas pré-natais $(\mathrm{n}=935)$} \\
\hline 0 & 82 & 8,8 & 52,4 & 0,96 & 0,$77 ; 1,20$ \\
\hline 1a 3 & 104 & 11,1 & 51,9 & 0,95 & 0,$77 ; 1,16$ \\
\hline 4 a 6 & 291 & 31,1 & 50,2 & 0,92 & 0,$79 ; 1,05$ \\
\hline 7 ou mais & 458 & 49,0 & 54,8 & 1 & \\
\hline \multicolumn{6}{|c|}{ Período de realização de exame anti-HIV (n=906) } \\
\hline Não fez exame anti-HIV & 193 & 21,3 & 52,8 & 0,97 & 0,$81 ; 1,16$ \\
\hline Primeiro trimestre da gravidez & 248 & 27,4 & 54,4 & 1 & \\
\hline Segundo trimestre da gravidez & 312 & 34,4 & 49,4 & 0,91 & 0,$77 ; 1,06$ \\
\hline Terceiro trimestre da gravidez & 153 & 16,9 & 54,2 & 1,00 & 0,$83 ; 1,20$ \\
\hline \multicolumn{6}{|l|}{ Resultado do exame anti-HIV ( $\mathrm{n}=733)$} \\
\hline Negativo & 585 & 79,8 & 52,6 & 1 & \\
\hline Não sei & 148 & 20,2 & 50,0 & 0,95 & 0,$79 ; 1,14$ \\
\hline
\end{tabular}


Tabela 3. Características de assistência ao parto de mães submetidas ao teste rápido anti-HIV em Hospitais Amigos da Criança segundo análise bivariada em relação à não amamentação do recém-nascido na primeira hora de vida. Rio de Janeiro, RJ, 2006

\begin{tabular}{|c|c|c|c|c|c|}
\hline \multirow{2}{*}{ Variável } & \multicolumn{2}{|c|}{ Freqüência } & \multicolumn{3}{|c|}{ Não-amamentação na primeira hora } \\
\hline & $\mathrm{n}$ & $\%$ & $\%$ & RR & IC 95\% \\
\hline \multicolumn{6}{|c|}{ Sabe se fez teste rápido anti-HIV (n=944) } \\
\hline Sim & 708 & 75,0 & 56,5 & 1 & \\
\hline Na dúvida/não & 236 & 25,0 & 40,7 & 0,72 & 0,$61 ; 0,85$ \\
\hline \multicolumn{6}{|c|}{ Resultado do teste rápido anti-HIV (n=943) } \\
\hline Negativo & 442 & 46,9 & 55,2 & 1 & \\
\hline Não sei & 501 & 53,1 & 44,8 & 0,72 & 0,$64 ; 0,81$ \\
\hline \multicolumn{6}{|c|}{ Quando soube resultado do teste rápido $(n=935)$} \\
\hline Antes do parto & 146 & 15,6 & 47,3 & 1 & \\
\hline Não soube & 501 & 53,6 & 44,3 & 0,94 & 0,$77 ; 1,14$ \\
\hline Depois do parto & 288 & 30,8 & 69,4 & 1,47 & 1,$22 ; 1,77$ \\
\hline \multicolumn{6}{|l|}{ Tipo de parto $(n=944)$} \\
\hline Normal & 637 & 67,5 & 47,9 & 1 & \\
\hline Cesáreo & 307 & 32,5 & 62,2 & 1,30 & 1,$15 ; 1,46$ \\
\hline \multicolumn{6}{|c|}{ Mãe queria amamentar ao nascimento $(n=940)$} \\
\hline Sim/mais ou menos & 542 & 57,4 & 46,3 & 1 & \\
\hline Não & 402 & 42,6 & 60,9 & 1,32 & 1,$17 ; 1,48$ \\
\hline \multicolumn{6}{|c|}{ Mãe refere que profissionais a escutam $(n=944)$} \\
\hline Sim & 719 & 76,2 & 50,1 & 1 & \\
\hline Mais ou menos/Não & 225 & 23,8 & 60,4 & 1,21 & 1,$06 ; 1,37$ \\
\hline \multicolumn{6}{|l|}{ Hospital $(n=944)$} \\
\hline 1 & 387 & 41,0 & 85,5 & 1 & \\
\hline 2 & 275 & 29,1 & 24,0 & 0,28 & 0,$23 ; 0,35$ \\
\hline 3 & 215 & 22,8 & 29,3 & 0,34 & 0,$28 ; 0,42$ \\
\hline 4 & 32 & 3,4 & 15,6 & 0,18 & 0,$08 ; 0,41$ \\
\hline 5 & 35 & 3,7 & 88,6 & 1,04 & 0,$91 ; 1,17$ \\
\hline
\end{tabular}

Foram submetidas a parto cesariano $32,5 \%$ das mães. Referiram que não queriam amamentar na sala de parto $42,6 \%$ das mães, principalmente por considerarem que o hospital é que deveria definir esta rotina, e em segundo lugar porque a condição do bebê não era favorável. A maior parte dos partos se concentrou em três hospitais, sendo observada uma grande discrepância na prevalência do desfecho entre eles, variando de $15,6 \%$ de não-amamentação na primeira hora de vida a $88,6 \%$. Consideraram que não foram escutadas pelo hospital $23,8 \%$ das mães. Todas as características da assistência hospitalar se mostraram significativamente associadas à não-amamentação na primeira hora de vida, em pelo menos uma de suas categorias (Tabela 3).

Após ajuste pelo modelo multinível, o recebimento do resultado do teste rápido anti-HIV após o parto dobrou o risco da não-amamentação na primeira hora de vida.
Outros fatores que contribuíram para postergar a primeira mamada foram: cor não branca, renda materna de um salário mínimo ou menos, parto cesáreo, mãe não querer amamentar o bebê ao nascimento e mãe referir que a equipe hospitalar não a escutava. O desconhecimento da realização do teste rápido anti-HIV pela mãe se mostrou um fator de proteção (Tabela 4).

\section{DISCUSSÃO}

No presente estudo, mais de um quarto $(28,5 \%)$ das parturientes foram submetidas ao teste rápido anti-HIV, proporção semelhante à encontrada em pesquisa realizada em 30 maternidades brasileiras $(28,1 \%){ }^{a}$ Em 2006, o Ministério da Saúde preconizava que o teste rápido anti-HIV fosse realizado naquelas parturientes que, não tendo sido testadas durante o pré-natal,

a Santos EM, Westman S, Reis AC, Alves RG. Avaliação do grau de implementação do programa de controle de transmissão vertical do HIV em maternidades do "Projeto Nascer", 2007 [citado 2009 nov 21]. Brasília; 2007. Disponível em: http://www.aids.gov.br/data/documents/ storedDocuments/\%7BB8EF5DAF-23AE-4891-AD36-1903553A3174\%7D/\%7B3F5A1D6B-09D0-4450-9060-2B5241E5F16B\%7D/ Avaliacao $\% 20$ Grau $\% 20$ Implementacao\%20Projeto\%20Nascer.pdf 
Tabela 4. Modelo multinível log log complementar de determinantes da não amamentação do recém-nascido na primeira hora de vida entre mães submetidas ao teste rápido anti-HIV em Hospitais Amigos da Criança. Rio de Janeiro, RJ, 2006.

\begin{tabular}{|c|c|c|}
\hline Variável & $\mathrm{RR}$ & IC $95 \%$ \\
\hline \multicolumn{3}{|l|}{ Cor da pele } \\
\hline Branca & 1 & \\
\hline Não branca & 1,40 & 1,$12 ; 1,74$ \\
\hline \multicolumn{3}{|l|}{ Renda materna } \\
\hline Mais que 1 salário mínimo & 1 & \\
\hline Mãe não tem renda & 1,23 & 0,$97 ; 1,56$ \\
\hline Um salário mínimo ou menos & 1,56 & 1,$21 ; 2,00$ \\
\hline \multicolumn{3}{|l|}{ Sabe se fez teste rápido anti-HIV } \\
\hline Sim & 1 & \\
\hline Na dúvida/Não & 0,72 & 0,$55 ; 0,94$ \\
\hline \multicolumn{3}{|l|}{ Resultado do teste rápido anti-HIV } \\
\hline Negativo & 1 & \\
\hline Não sei & 0,94 & 0,$54 ; 1,65$ \\
\hline \multicolumn{3}{|c|}{ Quando soube resultado do teste rápido } \\
\hline Antes do parto & 1 & \\
\hline Não soube & 1,03 & 0,$56 ; 1,90$ \\
\hline Depois do parto & 2,06 & 1,$55 ; 2,75$ \\
\hline \multicolumn{3}{|l|}{ Tipo de parto } \\
\hline Normal & 1 & \\
\hline Cesáreo & 1,70 & 1,$40 ; 2,07$ \\
\hline \multicolumn{3}{|l|}{ Mãe queria amamentar ao nascimento } \\
\hline Sim/Mais ou menos & 1 & \\
\hline Não & 1,42 & 1,$18 ; 1,72$ \\
\hline \multicolumn{3}{|c|}{ Mãe refere que profissionais a escutam } \\
\hline $\operatorname{Sim}$ & 1 & \\
\hline Mais ou menos/Não & 1,34 & 1,$08 ; 1,68$ \\
\hline
\end{tabular}

autorizassem sua realização após aconselhamento pela equipe de saúde. ${ }^{\mathrm{a}}$ Aconselhamento refere-se à troca de informações sobre os benefícios do diagnóstico precoce para o controle da infecção materna, para a prevenção da transmissão vertical e para a compreensão dos possíveis resultados do teste anti-HIV e seu impacto na vida da mulher. ${ }^{\mathrm{b}}$ No entanto, das 955 mulheres submetidas ao teste rápido entrevistadas, 585 (61,3\%) já dispunham de um resultado negativo do pré-natal. Além de o teste rápido anti-HIV ter sido realizado em quase dois terços das entrevistadas sem indicação, segundo as normas da época, muitas vezes foi praticado sem aconselhamento e sem consentimento da mulher, uma vez que $25 \%$ delas sequer tinham conhecimento da sua realização.

Os hospitais nos quais foi conduzida a pesquisa cumprem os "Dez Passos para o Sucesso do Aleitamento
Materno". ${ }^{17}$ Neles, espera-se que pelo menos $80 \%$ das mães com parto normal e 50\% daquelas submetidas ao parto cesáreo amamentem na primeira hora de vida. ${ }^{22}$ No entanto, no presente estudo, nem a metade $(47,5 \%)$ das mães submetidas ao teste rápido anti-HIV amamentaram seus filhos na primeira hora de vida, prevalência esta apenas um pouco superior à estimada pela Pesquisa Nacional de Demografia e Saúde da Criança e da Mulher para todo o Brasil (43,0\%) e para a Região Sudeste (37,7\%) no ano de 2006..$^{\mathrm{c}}$ Nos hospitais estudados, este evento teve múltiplas determinações, das quais a mais importante foi a mulher ter recebido o resultado do teste rápido após o parto.

Testes rápidos para a detecção de anticorpos anti-HIV são testes de triagem que produzem resultados em, no

${ }^{a}$ Ministério da Saúde. Portaria GM n ${ }^{\circ}$ 2104, de 19 de novembro de 2002. Institui no âmbito do Sistema Único de Saúde - SUS o Projeto Nascer-Maternidades. Diario Oficial Uniao. 2002 set 21;Seção1:48.

b Santos EM, Westman S, Reis AC, Alves RG. Avaliação do grau de implementação do programa de controle de transmissão vertical do HIV em maternidades do "Projeto Nascer", 2007 [citado 2009 nov 21]. Brasília; 2007. Disponível em: http://www.aids.gov.br/data/documents/ storedDocuments/\%7BB8EF5DAF-23AE-4891-AD36-1903553A3174\%7D/\%7B3F5A1D6B-09D0-4450-9060-2B5241E5F16B\%7D/

Avaliacao\%20Grau\%20Implementacao\%20Projeto\%20Nascer.pdf

c Ministério da Saúde. Pesquisa Nacional de Demografia e Saúde da Criança e da Mulher -PNDS-2006. Brasília; 2008. 
máximo, 30 min. ${ }^{a}$ No entanto, a rapidez do teste não garante que seu resultado esteja disponível à equipe da maternidade e à parturiente nesse mesmo prazo. No trabalho de campo foi observado que, nos cinco hospitais, o laboratório onde era realizado o teste rápido não se situava no mesmo andar da maternidade, o que dificultava a busca de seu resultado. A responsabilidade da busca do exame cabe ao profissional que o solicitou, ${ }^{\mathrm{b}}$ porém a grande demanda por testes rápidos e a sobrecarga de trabalho dos profissionais podem também ter dificultado a agilidade na obtenção dos resultados. Em pesquisa de avaliação do grau de implementação do Projeto Nascer, apenas $26 \%$ dos laboratórios entregavam os resultados dos testes rápidos em tempo hábil para a intervenção oportuna, tendo este indicador apresentado um grau de implantação crítico. ${ }^{c} \mathrm{O}$ Ministério da Saúde ${ }^{\mathrm{d}}$ afirma que cabe ao serviço proporcionar uma logística para que o resultado chegue em tempo hábil, e que o profissional de saúde não deve proibir o aleitamento materno para as mães cujo resultado do teste anti-HIV não esteja disponível. No entanto, observamos que não houve uma homogeneidade de conduta entre os hospitais pesquisados quanto a esta recomendação, o que contribuiu para a grande diferença na prevalência de não amamentação na primeira hora de vida encontrada entre eles.

A prevalência de teste rápido reagente de $0,8 \%$ foi o dobro da encontrada em Sergipe $(0,4 \%)^{11}$ e também da encontrada para o Brasil em $2004(0,4 \%),{ }^{18}$ possivelmente porque a pesquisa se restringiu a hospitais do sistema de gestação de alto risco.

É preconizada a administração de AZT intravenoso por no mínimo três horas antes do parto, a não realização de ruptura artificial de membranas e o parto cesáreo para as mães cujo resultado do teste rápido seja positivo. ${ }^{\mathrm{e}}$ Depreende-se, então, que a demora na entrega do resultado do teste rápido nestes hospitais tenha prejudicado não só a amamentação ao nascimento entre as mães soronegativas, como também a instituição de medidas profiláticas da transmissão vertical entre as soropositivas.

No modelo multivariado, o parto cesáreo aumentou o risco do desfecho em 70\%. Embora seja esperada uma menor proporção de amamentação ao nascimento entre as mães submetidas ao parto cesáreo, as proporções encontradas nesta clientela de mães submetidas ao teste rápido anti-HIV (52,1\% para parto normal e $37,8 \%$ para parto cesáreo) ainda estão distantes do preconizado. ${ }^{22}$ Outra variável associada ao desfecho foi a mãe referir que não queria amamentar ao nascimento. No entanto, ao serem analisados os motivos pelos quais a mãe não o desejava, verificou-se que a principal razão foi a mãe considerar que esta rotina deveria ser definida pelo hospital. Esta alegação sugere que a mulher abdica de seu desejo próprio em detrimento das rotinas hospitalares. Nos hospitais estudados, a maior parte das mães $(76,2 \%)$ sentiu-se ouvida sobre si mesma e o bebê. No entanto, não se sentir ouvida aumentou em $34 \%$ o risco da não-amamentação na primeira hora de vida.

Foi encontrado um sobre-risco de 56\% para a não-amamentação na primeira hora de vida entre as mães com renda materna igual ou inferior a um salário mínimo, e de $40 \%$ entre aquelas de cor não branca, pois as mães mais pobres possivelmente foram vistas pelos profissionais de saúde como clientela de risco. Pode-se supor que o aconselhamento foi mais praticado entre mães consideradas pelos profissionais de saúde como sendo de risco, para as quais poderia haver maior precaução em relação à amamentação em caso de status indefinido. $\mathrm{O}$ fato de a mãe não ter conhecimento da realização do teste rápido anti-HIV pelo hospital mostrou ser um fator de proteção para o desfecho.

Foi observado ainda, na análise multivariada, que o desconhecimento do resultado do teste rápido anti-HIV no momento da entrevista não se manteve associado ao desfecho. As mulheres que sequer tinham conhecimento da realização do teste rápido, provavelmente não foram identificadas pelos profissionais de saúde como pertencentes a grupos de risco, e sua submissão ao teste rápido pode ter sido um mero procedimento de rotina.

Algumas limitações devem ser consideradas. Só foi possível determinar o momento do resultado do teste rápido anti-HIV em menos da metade das mulheres devido à ausência de registro no prontuário de informações que permitissem caracterizar este momento e à ausência de comunicação pronta deste resultado às mães. Há também potencial viés de memória no recordatório do tempo decorrido até a primeira mamada, uma vez que este dado foi estimado pelas mães. O treinamento criterioso das entrevistadoras pode ter contribuído para minorar este viés.

Ainda assim, foi possível identificar que o fator de risco mais importante para a não-amamentação na primeira

a Conselho Federal de Medicina. Resolução CFM nº 1665, de 7 de maio de 2003. Diario Oficial Uniao 2003; 3 jun, Sec 1:83-4

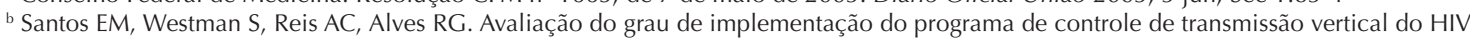
em maternidades do "Projeto Nascer", 2007. [citado 2009 nov 21]. Brasília; 2007. Disponível em: http://www.aids.gov.br/data/documents/ storedDocuments/\%7BB8EF5DAF-23AE-4891-AD36-1903553A3174\%7D/\%7B3F5A1D6B-09D0-4450-9060-2B5241E5F16B\%7D/

Avaliacao\%20Grau\%20Implementacao\%20Projeto\%20Nascer.pdf

c Ministério da Saúde. Secretaria de Vigilância em Saúde. Secretaria de Atenção à Saúde. Manual normativo para profissionais de saúde de maternidades da iniciativa Hospital Amigo da Criança. Referência para mulheres HIV positivas e outras que não podem amamentar. Brasília; 2004.

d Ministério da Saúde, Programa Nacional de DST e AIDS. Projeto Nascer. Brasília: Ministério da Saúde, 2003.

e Ministério da Saúde. Secretaria de Vigilância em Saúde. Programa Nacional de DST e AIDS. Recomendações para profilaxia da transmissão materno-infantil do HIV e terapia anti-retroviral em gestantes. Brasília; 2004. (Manuais, 46) 
hora de vida foi o recebimento do resultado do teste rápido após o parto, que implicou risco superior até ao parto cesáreo. Apesar de no presente estudo não ter sido realizada comparação de mulheres submetidas ao teste rápido com mulheres não submetidas, os resultados sugerem que o processo assistencial de realização do teste, por si só, já teve impacto sobre o início da amamentação. Foi observado que mesmo entre as mulheres com resultado do teste rápido anti-HIV antes do parto, a prevalência de amamentação ao nascimento foi abaixo da praticada em Hospitais Amigos da Criança. ${ }^{1}$ Pode-se supor que, quando o movimento de partos é intenso, mesmo estando disponível no prontuário, o resultado do teste rápido não é consultado pela equipe de sala de parto em momento oportuno.

Se estes resultados, consistentes em todos os hospitais estudados, foram obtidos em unidades que se caracterizavam pela promoção do aleitamento materno, poderia se pensar que nas demais maternidades públicas do Rio de Janeiro o quadro seria semelhante ou mais grave.

A partir de 2007, o Ministério da Saúde a passou a recomendar que, sempre que possível, além da realização de exame para detecção do HIV na primeira consulta de pré-natal, este exame fosse repetido no início do terceiro trimestre. Esta recomendação pode ter aumentado a demanda por testes rápidos, pela não disponibilidade do resultado do exame do terceiro trimestre na ocasião da internação para o parto. A testagem anti-HIV, mesmo em mulheres de baixo risco, está associada a altos níveis de ansiedade. ${ }^{2}$ Portanto, o ideal é que o teste seja realizado e seu resultado esteja disponível ainda na gravidez, não só pela maior chance de prevenção da transmissão vertical, ${ }^{\mathrm{b}}$ como também para que este processo não venha gerar ansiedade na mulher num momento sensível como o do parto e nascimento.

Recomendamos, assim, que os sistemas de referência e contra-referência dos serviços pré-natais com os laboratórios que realizam os exames sejam revistos, para que a realização e entrega dos resultados sejam agilizadas antes da internação para o parto. Os serviços hospitalares devem solicitar o teste rápido apenas quando indicado, incorporar a busca ativa dos resultados dos exames solicitados, e devolver este resultado prontamente às mulheres, pois a propriedade dos exames é da clientela. ${ }^{\mathrm{c}}$

É recomendável que o Sistema Único de Saúde, que disponibiliza o teste rápido, mais dispendioso, oriente as instituições na operacionalização adequada do teste, contribuindo para a prevenção da transmissão vertical e para o cumprimento do Passo $4^{22}$ na clientela de mulheres soronegativas testadas.

Recomendamos também que haja maior articulação entre as diversas políticas governamentais de assistência à mulher no período gravídico-puerperal. No presente estudo, foi possível constatar que o processo de implementação da prevenção da transmissão vertical do HIV no momento do parto, entendida como ação prioritária, vem ocorrendo sem uma adequada articulação com normas da Iniciativa Hospital Amigo da Criança, do programa de assistência pré-natal e da Política de Nacional de Humanização do Parto e Nascimento, previstas no Projeto Nascer. ${ }^{\mathrm{d}}$ Dentre os obstáculos a essa integração, encontram-se o grande volume de partos de algumas maternidades, a falta de capacitação e atualização permanente dos profissionais e as questões operacionais que envolvem a realização do teste anti-HIV e o aconselhamento das mulheres a ele submetidas.

Portanto, o cumprimento do Passo 4 da Iniciativa Hospital Amigo da Criança ${ }^{22}$ está diretamente associado ao desenvolvimento de ações que avancem na integralidade e transversalidade de políticas de assistência à mulher durante a gravidez, parto e puerpério, contribuindo para o fortalecimento de sua autonomia e melhoria da qualidade da atenção à saúde da mulher e da criança.

\footnotetext{
${ }^{a}$ Ministério da Saúde. Secretaria de Vigilância em Saúde. Programa Nacional de DST e Aids. Protocolo para a prevenção de transmissão vertical de HIV e sífilis: manual de bolso. Brasília; 2007. (B. Texto Básicos de Saúde -Manuais, 80).

${ }^{b}$ Ministério da Saúde. Secretaria Executiva. Coordenação Nacional de DST e AIDS. Recomendações para profilaxia da transmissão vertical do HIV e terapia antiretroviral em gestantes. Brasília; 2003. (Manuais, 46).

${ }^{c}$ Conselho Federal de Medicina. Resolução CFM nº 1498, de 10 de julho de 1998. Institui o Programa de Educação Continuada Excelência Médica, que contará com um Conselho Editorial escolhido na comunidade científica nacional ligada ao assunto. Diario Oficial Uniao. 1998 jul 27;Seção1:69.

${ }^{d}$ Ministério da Saúde. Portaria GM no 2104, de 19 de novembro de 2002. Institui no âmbito do Sistema Único de Saúde - SUS o Projeto Nascer-Maternidades. Diario Oficial Uniao. 2002 set 21;Seção1:48.
} 


\section{REFERÊNCIAS}

1. Araújo MFM, Schmitz BAS. Reassessment of Baby-friendly Hospitals in Brazil. I Hum Lact. 2007;23(3):246-52. DOI:10.1177/0890334407303899

2. Biehl J, Coutinho D, Outeiro AL. Technology and effect: HIV/AIDS testing in Brazil. Cult Med Psychiatry. 2001;25(1):87-129. DOl:10.1023/A:1005690919237

3. Carvalho RL, Krahe C, Farina G, Paula DO, Richetti N, Crossettti T. Teste rápido para diagnóstico da infecção pelo HIV em parturientes. Rev Bras Ginecol Obstet. 2004;26(4):325-8. DOI:10.1590/S010072032004000400010

4. Oliveira MIC, Camacho LAB. Impacto das unidades básicas de saúde sobre a prevalência de aleitamento materno exclusivo. Rev Bras Epidemiol. 2002;5(1):4151. DOI:10.1590/S1415-790X2002000100007

5. Dourado I, Veras MASM, Barreira D, Brito AM. Tendências da epidemia de Aids no Brasil após a terapia anti-retroviral. Rev Saude Publica. 2006;40(Supl):9-17.DOI: 10.1590/S003489102006000700003

6. Duarte G, Gonçalves CV, Marcolin AC, Paschoini MC, Quintana SM, Mussi-Pinhata MM. Teste rápido para detecção da infecção pelo HIV-1 em gestantes Rev Bras Ginecol Obstet. 2001;23(02):107-11. DOI:10.1590/S0100-72032001000200008

7. Dunn TDT, Newell ML, Ades AE, Peckham CS. Risk of human immunodeficiency virus type 1 transmission through breastfeeding. Lancet. 1992;340(8819):585-8. DOI:10.1016/0140-6736(92)92115-V

8. Edmond KM, Zandoh C, Quigley MA, AmengaEtego S, Owusu-Agyei S, Kirkwood BR. Delayed breastfeeding initiation increases risk of neonatal mortality. Pediatrics. 2006;117(3):e380-6. DOI:10.1542/peds.2005-1496

9. Goldstein H. Multilevel Statistical Models. 2.ed. London: Edward Arnold; 1995. (Kendall's Library of Statistics, 3).

10. Heinig MJ, Dewey KG. Health effects of breast feeding for mothers: a critical review. Nutr Res Rev. 1997;10(1):35-56. DOI:10.1079/NRR19970004

11. Lemos LMD, Gurgel RQ, Fabbro ALD. Prevalência da infecção por HIV em parturientes de maternidades vinculadas ao SUS. Rev Bras Ginecol Obstet. 2005;27(1): 32-6. DOI:10.1590/S010072032005000100007
12. McCullagh P, Nelder JA. Genelalized Linear Models. 2. ed. London: Chapman and Hall/CRC Press; 1989.

13. Martines JC, Ashworth A, Kirkwood B. Breast-feeding among the urban poor in southern Brazil: reasons for termination in the first 6 months of life. Bull World Health Organ. 1989;67(2):151-61.

14. Boletim Epidemiológico AIDS e DST. Brasília: Ministério da Saúde;2007;7(1).

15. Murray EK, Ricketts S, Dellaport J. Hospital practices that increase breastfeeding duration: results from a population-based study. Birth. 2007;34(3):202-11. DOI:10.1111/j.1523-536X.2007.00172.x

16. Righard L, Alade MA. Effect of delivery room routines on success of first breast-feed. Lancet. 1990;336(8723):1105-7. DOI:10.1016/01406736(90)92579-7

17. Saadeh R, Akré J. Ten steps to successful breastfeeding: a summary of the rationale and scientific evidence. Birth. 1996;23(3):154-60. DOl:10.1111/j.1523536X.1996.tb00476.x

18. Szwarcwald CL, Souza Jr PRB. Estimativa da prevalência de HIV na população brasileira de 15 a 49 nos, 2004. Bol Epidemiol AIDS-DST. 2006;3(1):11-5.

19. Tess BH, Rodrigues LC, Newell ML, Dunn DT, Lago TD. Breastfeeding, genetic, obstetric and other risk factors associated with mother-to-child transmission of HIV-1 in São Paulo State, Brazil. São Paulo Collaborative Study for Vertical Transmission of HIV-1. AIDS. 1998;12(5):513-20. DOI:10.1097/00002030199805000-00013

20. Veloso VG, Portela MC, Vasconcellos MTL, Matzenbacher LA, Vasconcelos ALR, Grinsztejn B et al. HIV testing among pregnant women in Brazil: rates and predictors. Rev Saude Publica. 2008;42(5):859-67. DOI:10.1590/S0034-89102008000500011

21. Victora CG, Smith PG, Vaughan JP, Nobre LC, Lombardi C, Teixeira AM, et al. Evidence for protection by breast-feeding against infant deaths from infectious diseases in Brazil. Lancet. 1987;8(8554):319-22. DOI:10.1016/S0140-6736(87)90902-0

22. World Health Organization, The United Nations Children's Fund. The global criteria for the WHO/ UNICEF Baby-Friendly Hospital Initiative. Geneva; 1992.

Pesquisa financiada pelo Conselho Nacional de Desenvolvimento Científico e Tecnológico (CNPq, Processo No: 403015/2005-7).

Trabalho apresentado no X Encontro Nacional de Aleitamento Materno, Belém, PA, em 23/5/2008. 